\title{
A Sustainable Application Based on Grouping Genetic Algorithm for Modularized Redesign Model in Apparel Reverse Supply Chain
}

\author{
Manoj Kumar Paras ${ }^{1,2,3}{ }^{\circledR}$, Lichuan Wang ${ }^{1, *}$, Yan Chen ${ }^{1}$, Antonela Curteza ${ }^{2}$, Rudrajeet Pal ${ }^{3}$ ([) \\ and Daniel Ekwall ${ }^{3,4}$ \\ 1 College of Textile and Clothing Engineering, Soochow University, Suzhou 215006, China; \\ manoj.kr.paras@gmail.com (M.K.P.); yanchen@suda.edu.cn (Y.C.) \\ 2 Faculty of Textile, Leather and Industrial Management, "Gheorghe Asachi” Technical University of Iași, \\ 700050 Iași, Romania; acurteza@gmail.com \\ 3 Faculty of Textiles, Engineering and Business, University of Borås, Allégatan 1, 50190 Borås, Sweden; \\ rudrajeet.pal@hb.se (R.P.); Daniel.ekwall@hb.se (D.E.) \\ 4 Supply Chain Management and Social Responsibility, Hanken School of Economics, Arkadiankatu 22, \\ 00100 Helsinki, Finland \\ * Correspondence: lcwang@suda.edu.cn; Tel.: +86-188-9658-8991
}

Received: 8 June 2018; Accepted: 21 August 2018; Published: 24 August 2018

\begin{abstract}
The scarcity of natural resources and the problem of pollution have initiated the need for extending the life and use of existing products. The concept of the reverse supply chain provides an opportunity to recover value from discarded products. The potential for recovery and the improvement of value in the reverse supply chain of apparel has been barely studied. In this research, a novel modularized redesign model is developed and applied to the garment redesign process. The concept of modularization is used to extract parts from the end-of-use or end-of-life of products. The extracted parts are reassembled or reconstructed with the help of a proposed group genetic algorithm by using domain and industry-specific knowledge. Design fitness is calculated to achieve the optimal redesign. Subsequently, the practical relevance of the model is investigated with the help of an industrial case in Sweden. The case study finding reveals that the proposed method and model to calculate the design fitness could simplify the redesign process. The design fitness calculation is illustrated with the example of a polo t-shirt. The redesigned system-based modularization is in accordance with the practical situations because of its flexibility and viability to formulate redesign decisions. The grouping genetic algorithm could enable fast redesign decisions for designers.
\end{abstract}

Keywords: reuse design; upcycling; modularization; garment industry; sustainability

\section{Introduction}

The process of redesigning an existing product is considered to be relatively less important than manufacturing new products. Redesigning is a common strategy adopted by companies to develop a second-generation product from the existing unsold or lesser demanded products [1]. Second-generation products are made by changing the product form, enhancing the product function, or improving the way to use the product [2]. Product redesign by transforming its physical form is one of the easiest and common methods that does not need an advanced technology or a huge investment. However, it is difficult to achieve a satisfactory aesthetic and functional product by randomly changing the product form [1]. 
Products may need to be redesigned after use in some situations. There could be several reasons for redesign, such as extending lifespan, reducing environmental impacts, or customers may have become fed up with the look of the product and want to change the look. In today's scenario, a consumer owns and uses multiple products, hence the redesign becomes important [3]. However, the current redesign process fails to utilize innovative methods. Most of the redesign activities are undertaken to extract/replace useful parts or change attributes/structures of the products [4]. Baxter [5] has identified that the success of the product design with a well-defined process and specifications is three to four times higher than the random method.

The present work utilizes a genetic algorithm and modular product design techniques to redesign products. Modular product design is an important strategy to handle the components of end-of-use and end-of-life products [6]. The extracted components or parts of products are reassembled and redesigned with the genetic algorithm using design and industry-specific domain knowledge. A genetic algorithm is an evolutionary computation technique to achieve high design fitness in the absence of explicit function [7]. Design fitness is also calculated to measure the redesign fitness. Considering a case study from the garment industry, the study will illustrate the model development.

The paper is organized as follows: Section 1 introduces the redesign process for garments. Section 2 uses a genetic algorithm and modular design techniques to develop the redesign method. Section 3 aims to develop a redesign model and a numeric method to calculate the design fitness for garments. Section 4 presents a case study to illustrate the garment redesign process of a Stockholm, Sweden-based company, and Section 5 discusses the findings from the redesign model and case study, and provides the conclusion.

\section{Redesign Technique in Upcycling}

There are different techniques and reasons to redesign a product. Some of the common reasons to redesign the products are to overcome design faults, reduce the manufacturing cost, create a product family, or develop a new product from two or more different products [3]. The particular design of a product can be modified or redesigned to minimize the production cost. In today's scenario, the cost reduction and the environmental impact are generally considered and evaluated throughout the product life cycle [3]. Efforts are made to redesign the product by identifying weak spots in the current design and modifying the same to maximize the end-of-life value [8]. Products are designed to fulfill customers as well as environmental requirements. Component analysis for a product is performed to determine the overall product value and component value, on the basis of which the components are redesigned to reduce the negative environmental impacts [9]. To reduce the overall cost and increase the performance, a component can be changed with a low cost and better performing component [10]. The product can be redesigned into modules for ease of maintenance, reuse, and recycling [11]. The modular product innovation has stimulated the redesign ability to minimize the cost and maximize the use.

\subsection{Modularization Process for the Redesign}

The concept of modular redesign can be extended to product family creation where a component or group of components (i.e., subsystem) can be designed to be implemented across the range of products [12]. Redesigning a component or subsystem for a product family simplifies the product designing process, maximizes the use of a components/subsystem, and minimizes the cost [3]. The process of creation of a component or subsystem for the product family consists of breaking down product design into modules and selecting common components to reuse across a variety of products [13]. Generally, the modular component structure is recommended for low-volume and customized products to minimize the overall cost. The modular redesign process can convert heterogeneous components to a homogenous set of components. The product components with less or no commonality can be grouped according to functionality for multiple uses [14]. Modular-based redesigning reduces the cost, and increases the profit without changing the structure of the products. 
The modularization technique is a widely useful and accepted method for product design and manufacturing to improve the performance of a supply chain. It is wise to reduce the large system into small subsystems to cope with the rising complexity of product design and manufacturing. Under the concept of modularization, the small and simple processes are also clustered into subassemblies for ease of handling. In the modular structure, a subpart is treated as a functional unit with few interactions between subparts. There are numerous benefits of modularizations, such as improvement in the manufacturing efficiency, the supply chain efficiency, and overall effectiveness. The modularization technique has evolved over a period of time, and can be categorized into three categories: (1) matrix function classification, (2) cluster graph math artificial genetic classification, and (3) sustainability classification [6]. The matrix method uses the clustering technique to sort components into different product modules. The function method breaks down complicated functions to generate subsystems or modules [15]. The clustering method helps in the formation of group components on the basis of similarities and differences. The graphical method uses a matrix technique to sort and create a module. Mathematical programming uses operation research techniques to form groups or modules. The artificial intelligence technique uses computer science knowledge to cluster the components. Similarly, genetic and heuristics methods are also used for module forming [16]. The social, economic, and environmental aspects of sustainability can also be considered to create a module or classification [6].

The concept of a modularized system draws its foundation from the intelligent design system. This enables the manufacturing process to achieve high quality, and the system of modularity also provides flexibility for the disassembly and redesign after the product end-of-life [17]. Discarded product after the end-of-life can be broken down into parts or components to encompass the unused value that is associated with it. There are different end-of-life options available for the discarded product such as reuse, remanufacturing, upcycling, downcycling, landfill, and incineration [18]. The concept of modularization maximizes the use of a part or set of parts that are extracted from a discarded product. The module structure enhances the performance and sustainability [6]. The modularization design strategy standardized the design process for mass customization. Under the concept of modularization, one part can be fabricated for more than one product. This strategy has been successfully adopted and implemented in the electronics industry. Modularization is the technique for the product and process redesign formalization to reduce the variations among parts or components [19]. An intelligent garment design process on the basis of the modularization technique has been used by Zhou et al. [20] for its flexibility and user-friendly design process.

\subsection{Grouping Genetic Algorithm}

At present, the market is flooded with thousands of products where the designs of these products are developed through rigorous and innovative product development processes. In several instances, the design of the product doesn't fulfil the requirements; therefore, the products or its components are required to be redesigned to meet requirements. Li et al. [4] have proposed an attribute-based model to resolve the differences between product design and its functional or aesthetic requirements. The design issue is addressed by changing the components, attributes of components, or the structure of the product design. In the case-based redesign approach, the design information from the previous stages can be used to achieve a new design [21,22]. The flexible or self-redesign system is another holistic approach to address the fast-changing requirements of consumers. Kasarda et al. [23] have developed a design method for adaptation to self-adjust or self-redesign based on the attributes' parameters. An existing product can be modified on the basis of the design attributes, and their value, to address the design and functional differences.

Genetic algorithm (GA) is an artificial intelligence and mathematical optimization technique that has repeatedly been used to solve a problem more quickly when classic methods take time to find solutions. GA is inspired by the biological mechanism of natural evolution from one generation to another to generate a best possible solution. The new solution is obtained through three steps 
i.e., selection, crossover, and mutation [24]. At the selection phase, two parents are selected based on their suitability or fitness. In the next step, selected parent crossover is reproduced to obtain a new individual. During crossover, half of two or more parents are swapped to create a new solution. The third step is a mutation to bring small tweaks or improvements to the new individual. Mutation is applied to bring diversity to the existing population [25]. Genetic algorithm methods can be used for grouping an irregular shape at an optimal level [26]. It is difficult to achieve the best grouping through the manual method involving the hit and trial approach. The heuristic approach of the genetic algorithm can achieve the best fit to the group [27,28]. In the grouping genetic algorithm (GGA), the user can achieve fitness by grouping the components through an iterative process. Thus, the GGA can provide better design; therefore, the GGA is better than the GA tool [7]. In recent days, the approach of the genetic algorithm can also be used in the textile industry to solve various problems [29,30]. Yang and Lin [31] have used a genetic algorithm in the footwear industry to solve pattern problems.

\section{Modularization Redesign Method Based on Grouping Genetic Algorithm}

The present work aims to develop a redesigned model by considering three different problems: (i) the modular method for the extraction of components, (ii) the attributes-based clustering method, and (iii) the calculation of the design fitness of components. In this paper, the modular redesign method proposed by Farrell and Simpson [32] is employed to extract components from reference end-of-life or end-of-use products. The components are extracted to maximize the commonality to simplify the redesign process. Mutingi et al. [33] have proposed that the number of modules that a product can have is dependent upon the total number of components of the product. A minimum number of useful components that can be extracted from a product is set to be 1 , whereas the maximum number of components that can be extracted from a product can be represented by Formula (1):

Number of useful components that can be extracted $\leq \sqrt{ }$ Total number of components of the product

Each of the extracted components are defined by their attributes such as length, composition, shape, and color. A numeric value is assigned to the attributes for dimension (length and width) and the color type of extracted parts. Different kinds of parts can be extracted from products that can be categorized based on their significance in relation to important and less important parts. During the extraction of parts from used or discarded products, the following rules can be deployed:

1. The extraction of parts should be done at a medium level. Otherwise, connections and design could be complicated due to too many parts.

2. Decisions about important and less important parts should be made on the basis of its use in the target products. Important parts are those parts that can be used for making upcycled or higher valued products.

3. Less important parts could be used for making downcycled or lower value products such as accessories.

4. Extracted parts will be used for redesign and reconstruction. Therefore, the domain knowledge of product production is suggested to make subjective decisions.

On the basis of the above rules, the extracted parts of products can be categorized and subcategorized, as illustrated in Table 1. The details of extracted parts are determined by key design and composition attributes that can vary from one product to another.

Each part has certain characteristics and an associated value to its attributes. Change in the type of the attribute will provide different parts. The products that are selected for redesign could consist of different parts. All of the parts of a used product cannot be useful. Some of the parts will be useful, while others may not be suitable for a redesign. 
Table 1. Characteristics of modular parts.

\begin{tabular}{ccc}
\hline Part Type & Coding & Part Name \\
\hline \multirow{5}{*}{ Important } & I1 & Product 1 front \\
& I2 & Product 1 back \\
& I3 & Product 1 other parts 1 \\
& I4 & Product 1 other parts 2 \\
& I5 & Product 2 front \\
I6 & Product 2 back \\
& I7 & Product 3 body \\
I8 & Product 4 body \\
\hline \multirow{4}{*}{ Less important } & L1 & Common accessories 1 \\
& L2 & Common accessories 2 \\
& L3 & Common accessories 3 \\
& L4 & Other accessories \\
\hline
\end{tabular}

The extracted module can be grouped with the help of GA as proposed by Gu and Hashemian [34]. However, the number of components and value of attributes need to be determined at the beginning. To overcome this limitation, Falkenauer [35] has proposed GGA to reselect or replace components if a particular module or components are not found to be not suitable to achieve the desired target products. The repetition of these crossover steps can generate new offspring. If suitable modules cannot be found to achieve the desired design, then, on the basis of GGA, the steps can be repeated as depicted in Figure 1:

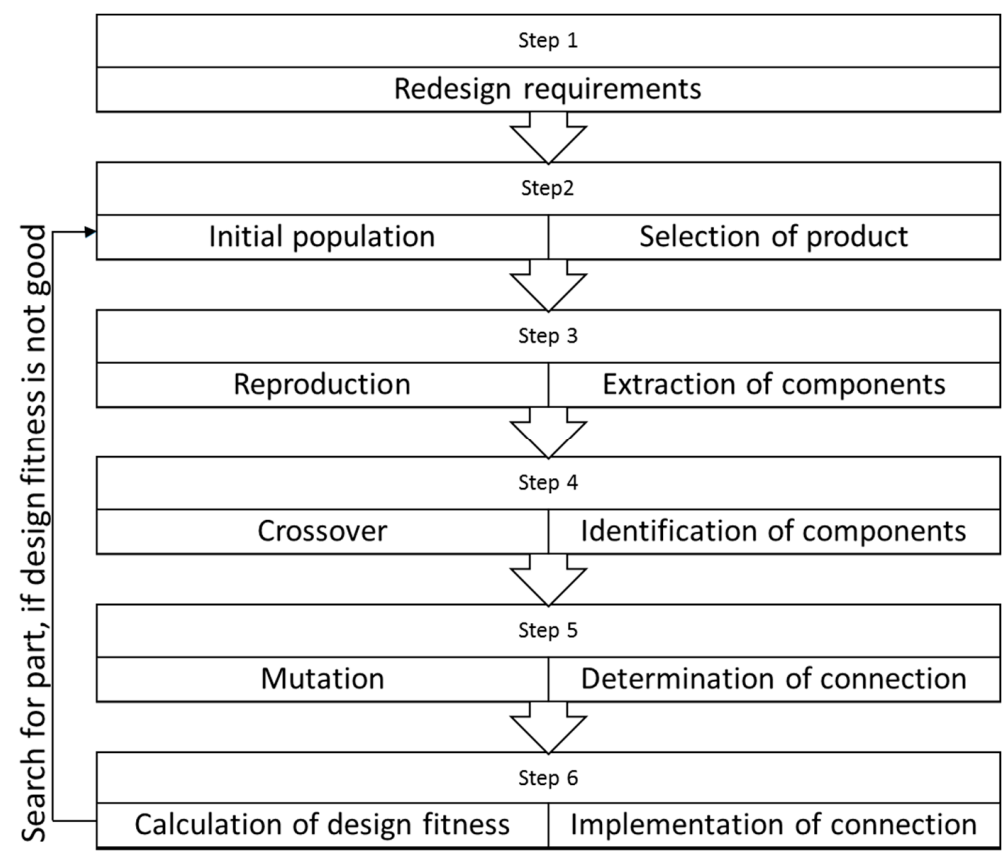

Figure 1. Grouping genetic algorithm for the modularized redesign.

Design fitness can be achieved by adopting three mutation strategies suggested by De Lit et al. [36]. The parts can be randomly moved to find the most suitable parts as per the requirement of target products. To achieve this, a new part could be added to existing products, or maybe some parts can also be deleted. Further, suitable parts can also be found irrationally with the help of a heuristic algorithm. The mutation of selected parts will be performed only if a certain degree of similarities are achieved between selected parts and target product design. This can help to achieve an optimal design value for the target products. 
Two or more reference products can be combined to develop new products. The redesigned model uses a modular redesign technique and the modular design principle to extract, reconstruct, or combine two products. This improves the quality and reduces time over traditional manual redesign techniques. The redesigned model consists of six steps, as demonstrated below:

Step 1: Redesign requirements identification for target products: The redesign of a new product depends upon the clear understanding of requirements. A designer should have a clear understanding of current market trends and customer requirements.

Step 2: Selection of end-of-use (life) products for reference: After the identification of redesign and customer requirements, the next step is the selection of used products. Two or more distinct or similar products can be selected to combine into a single new product.

Step 3: Extraction of components from reference products: After the selection of reference products, the next step is to identify the usable components from the reference products. Components are extracted and stored on the principle of modular design, as demonstrated in Equation (1).

Step 4: Identification of components for target products: The next step involves the identification of components for the target products. The components are selected on the basis of the redesign requirements conceived for the target products.

Step 5: Connection of components to develop target products: Identified components are connected to each other to achieve the desired redesign requirements. Parts are connected so that at the connection site, the attributes and the value of the attributes should be similar. The connection between two parts is based on the type of joint, the machine used, and the duration for which the machine is used. The numerical value is assigned to each connection and component attribute for design fitness calculation.

Step 6: Calculation of redesign fitness for connection: If $M u_{A}$ and $M u_{B}$ are two parts, then the design fitness or connection intensity can be defined as $C I_{A B}$, where $T_{n}$ can be defined as the total connection intensity for new product $M_{n}$. Practically, two parts are connected by multiple operations or connections that can be denoted by $C_{n}$. The accumulated connection intensity can be represented as follows:

$$
T_{n}=\sum_{M u_{A} \in M_{n}} \sum_{M u_{B} \in M_{n}} C I_{A B} / C_{n}
$$

The accumulated connection intensity $C I_{\text {total }}$ is constant, because of the total number of connection or operations; $C_{n}$ in any product $M_{n}$ is fixed. The goal of the grouping genetic algorithm (GGA) is to optimize the connection intensity or design fitness. The garment redesign method is selected to validate the genetic grouping-based redesign strategy.

\section{Redesign for Garments}

Redesign is a process that adds value to discarded or used garments. The original idea of the garments' redesign was based on the technique of product design [37]. The degree of redesign can differ from minor changes to the overall transformation of the garments. A minor change could be possible by adding decorative trims, new embroidery, or print [38]. The overall transformation could be achieved by changing the style or pattern of the garments. For example, denim trousers could be transformed into lady jackets by creating a new pattern and reconstruction. The possibility of redesigning a garment can be decided based on its structure, fabric, and quality [39]. The used or discarded garments need to be selected on the basis of various parameters such as durability, deconstruction complexity, and current trends [40]. Design thinking is an iterative process that consists of inspiration, ideation, and implementation. Any design intervention generally oscillates between the inspiration and ideation stages [41]. The inspiration and ideation for redesign can be clubbed together, as separate inspiration and ideation required for each individual product. Notwithstanding, the implementation steps can be broken down into reconstruction and fitting. By and large, the process 
of redesign can be categorized into the selection, ideation, reconstruction, and fitting stages as depicted in Figure 2.

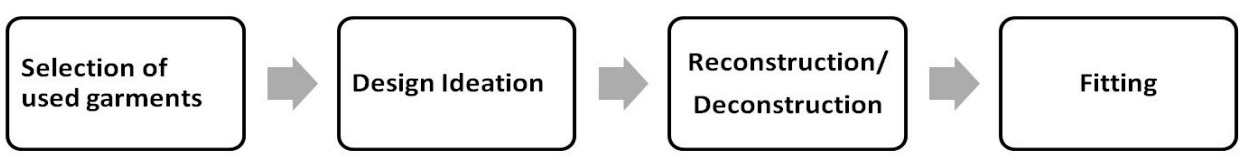

Figure 2. Redesign process (adapted from Paras and Curteza [40]).

The redesign process takes enormous time to find the right combination while transforming particular styles. The confinement of redesign can be manifested in terms of variation in the size, pattern, fabric, and color of the selected products [42]. To illustrate, used garments that are bigger in shape or have an easy pattern can simply accommodate the new product design, and vice versa for the complex design and smaller size garments. Further, each fabric has different material properties and colors, which can be considered more constraints due to varied product selection. However, these restrictions can also be viewed as a prospect for the redesigning process. Put differently, the size and pattern of each fabric that is retrieved from the used product and is distinctive can provide a unique design solution for new and improved products [40]. In order to convert constraint into opportunity, the redesigning process requires meticulous skills to maintain precision [43]. Further, a significant amount of time is required to achieve the desired outcome. Each part of the used product is novel, which needs to be handled independently to achieve higher redesign ability. An inventive approach is likewise expected to handle the unpredicted quality and quantity of discarded products. It is difficult to run the continuous process of redesign with a restricted supply of input. In the fast-changing fashion world, the fashion of the earlier seasons provides a reference point, and hence becomes valuable for consideration. Therefore, it is important to retain product provenance [40]. This can be achieved by upcycling or redesigning old products with improved aesthetics or/and functional properties. The pictures in Figure 3 illustrate some of the redesigned garments.

The narrative of the upcycled product is another important factor that increases its saleability. The narrative of the product's previous life could be an important promotion strategy [42]. Deschamps et al. [44] have additionally featured the requirement for an upcycle brand to retain the history of the item. In the present work, a grouping genetic algorithm-based modularization process is applied to the modularized garment redesign model. The grouping genetic algorithm is used to create second-generation products by matching the attributes of the extracted garments' parts.
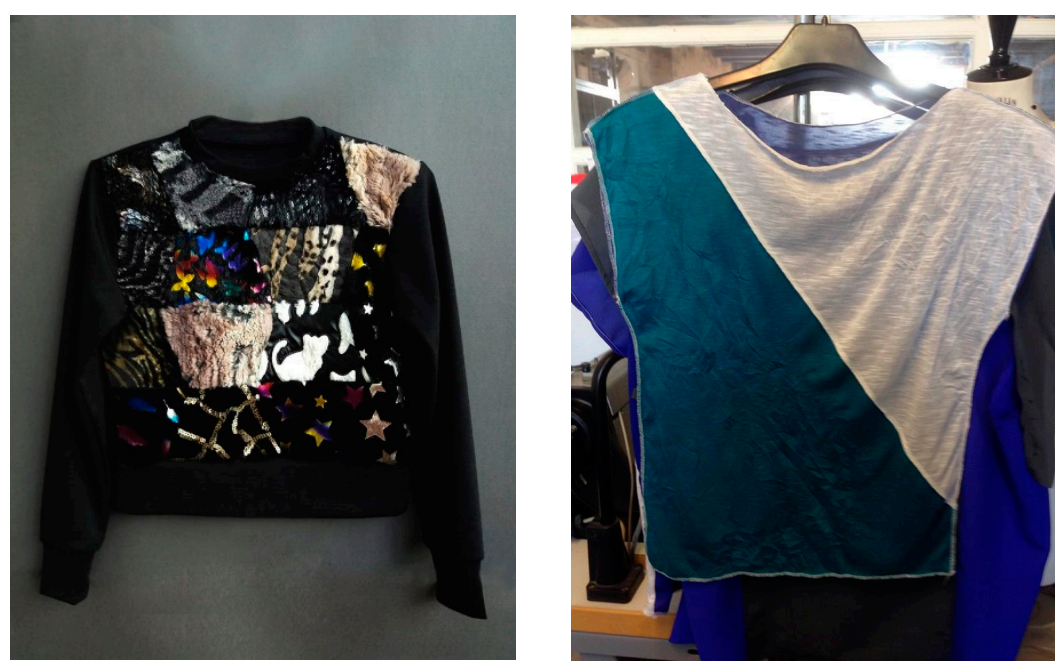

Figure 3. Cont. 

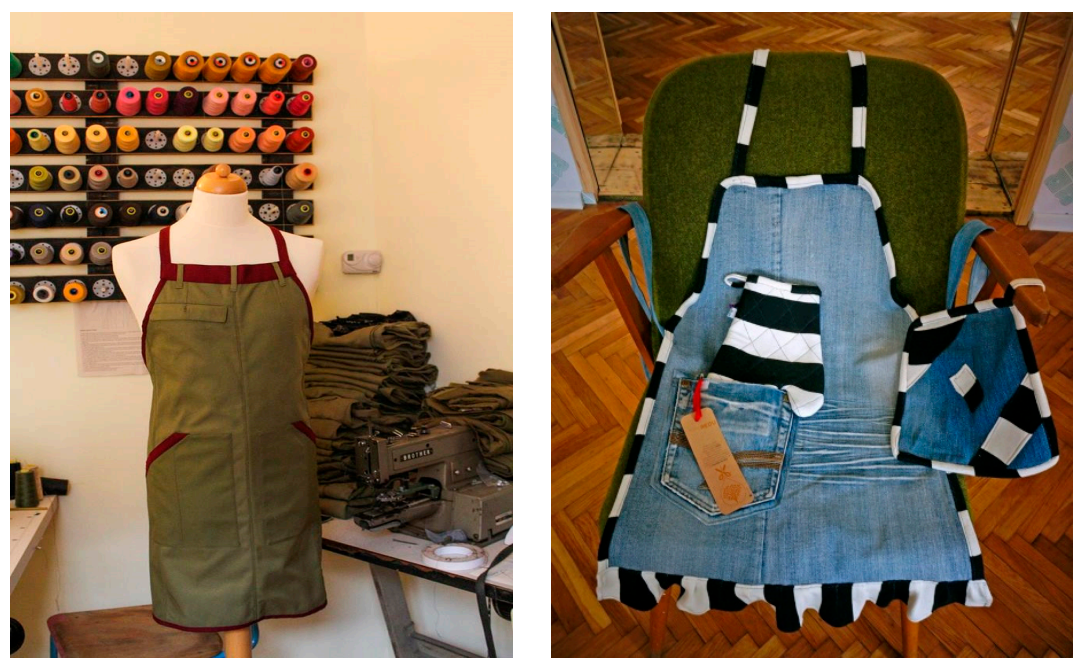

Figure 3. Examples of various garment redesigns (source: organization page on social media).

\subsection{Model for Garment Redesign}

This section is intended to develop a garment redesign model. A garment can be redesigned based upon the demand or need for products. The sketch, idea, or design of the target product can be developed to fulfill the functional and aesthetic design requirements. Used or old garments can be selected as a reference product to fulfill the requirements of the redesign garment. The reference garments are selected as reasonably close matches to the user and redesign garments' requirements. The usable parts from the reference garments can be extracted. Some of the extracted parts from different reference products could be common and can be stored together. A formal t-shirt or top consists of a 'front', 'back', 'sleeve', 'neck rib', and 'sleeve rib'. Similarly, the 'front', 'back', 'waistband', and 'pocket' can be extracted from a trouser or bottom, as illustrated in Figure 4.

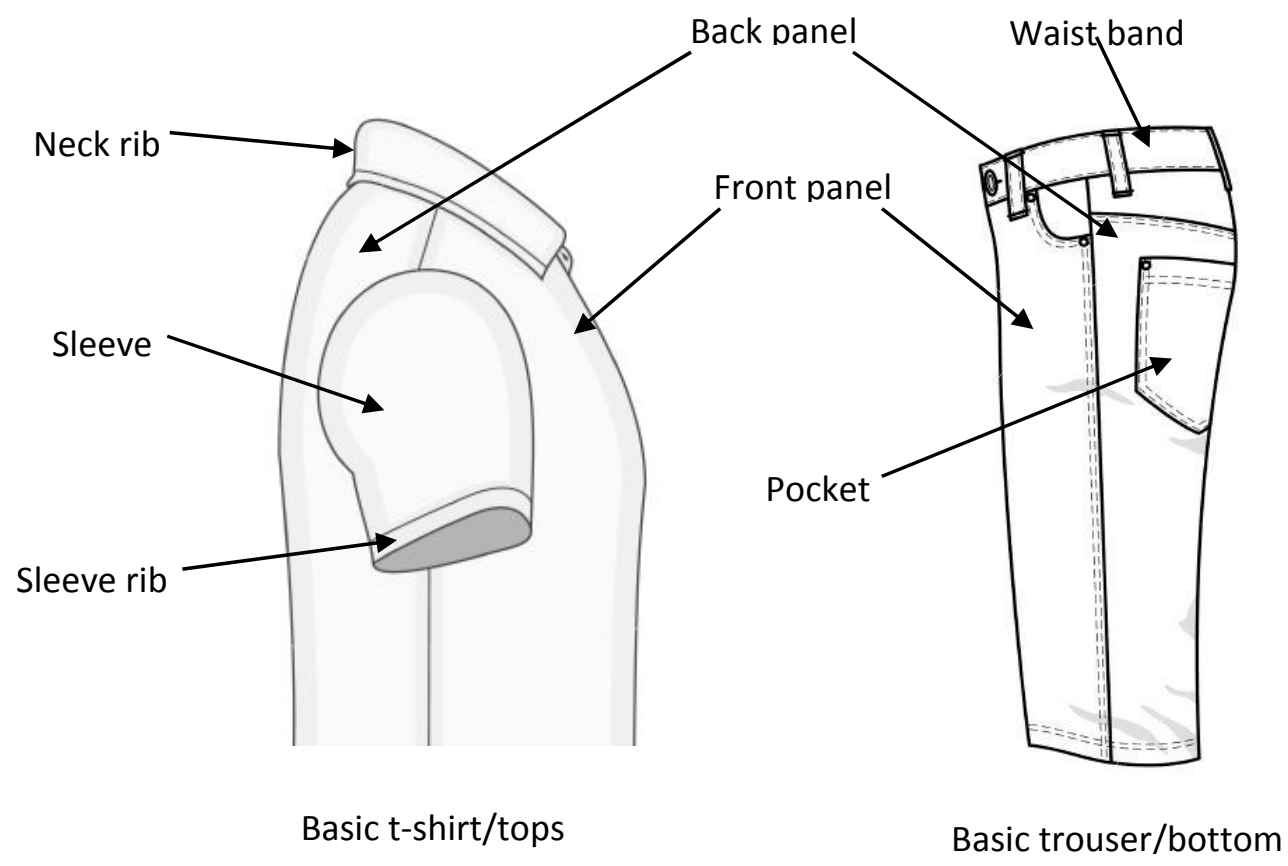

Figure 4. An illustration of reference/target garments.

Each extracted parts can be categorized based on the characteristics or attributes of the parts. For example the 'front' and 'back' panels of tops and bottoms can be stored in a separate place, 
respectively. Further, each part with the same characteristics or attributes can be subcategorized based on the attributes' value or dimension. Designers could attempt to develop target products for every possible design combination (i.e., crossover from the initial population) of extracted parts. Further, mutation operation could be performed to insert some additional features. During the redesign process, various kinds of ideas or developed designs can be stored in the reference design database for future use. Redesign garments (target products), used/old garments (reference products), and the design database (reference design) can interact with each other to optimize the redesign process. All three databases (i.e., design, extracted parts, and target products) could be updated every time after a successful redesign. This will enable strengthening the redesign process. Figure 5 represents a structured model for the garment redesign process.

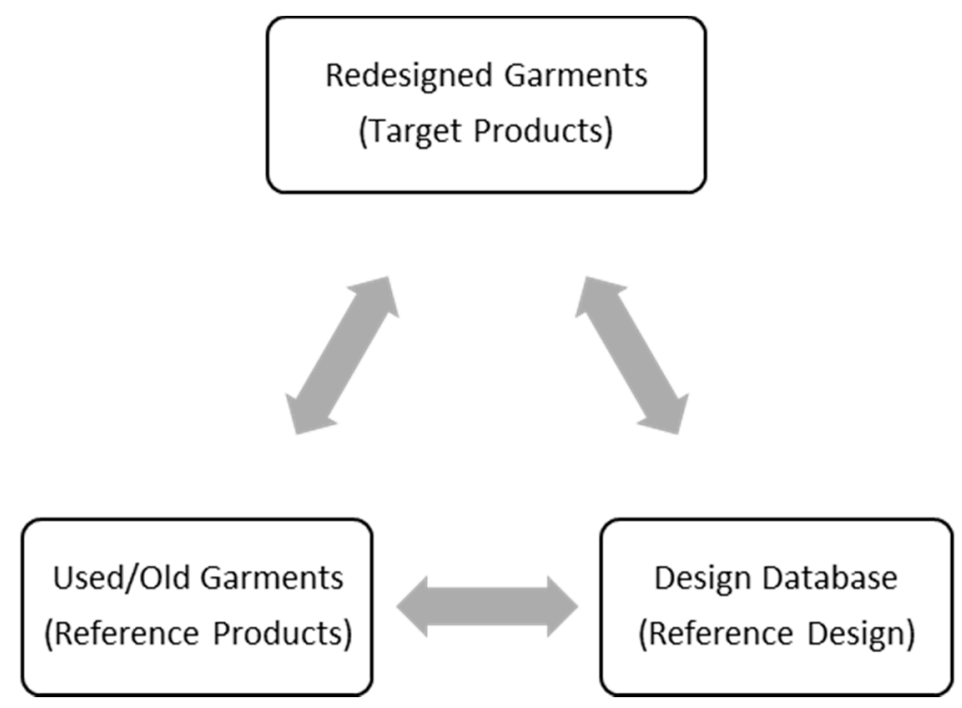

Figure 5. Modularized garment redesign model.

The next stage is to join parts after the extraction and identification of parts for the redesign product. Each part can be connected or joined with a different type of machine, seam, and stitch length. Design fitness or connection strength can be calculated to rate the redesign on the basis of the connection (machines, seam, stitch length) and part attributes. Numerical values help to quantify the design fitness or connection intensity.

\subsection{Calculation of Design Fitness}

The strength or intensity of the connection depends on different connection attributes such as the seam type (id), the machines used to stitch the seam, and the attributes $(T)$ and value $(V)$ of attributes such as the fabric composition, fabric color, and the junction/connection. The junction or connection attribute value can be defined in the terms of seam length or standard allowed minute (SAM)/standard minute value (SMV). A number between 0 and 100 is allocated for each attribute depending on the strength of its connection. The weightage of each of the junction's attributes is assigned on the basis of its importance for a connection, as proposed by Tseng et al. and Das et al. [24,45]. The weightage for seam type is assigned 30\%, machine type-35\%, seam length-15\%, and fabric attributes (such as color, composition etc.) $-20 \%$. These four attributes collectively decide the connection intensity (CI). A higher value of $\mathrm{CI}$ denotes better connection.

\subsubsection{Seam Type}

The seam type depicts the method to attach two parts. The attachments of two parts are strongest for the superimposed seam type, whereas the seam strength in the decorative seam is the least amongst all. Therefore, the highest connection intensity is assigned for the superimposed seam, while the lowest 
connection intensity is assigned to the decorative seam. Assumption has made for CI of different type of seam and illustrated in Table 2.

Table 2. Connection intensity of seam type.

\begin{tabular}{ccc}
\hline S.No. & Seam Type & Connection Intensity \\
\hline S1 & Decorative & 6 \\
S2 & Flat & 12 \\
S3 & Bound & 18 \\
S4 & Lapped & 24 \\
S5 & Superimposed & 30 \\
\hline
\end{tabular}

\subsubsection{Machine Type}

The strength of connection also depends on the machine that is used to attach two seams. For example, the single needle lockstitch machines can provide the strongest connection; hence, the highest connection intensity is assigned to a single needle lockstitch, while a lesser intensity is assigned to another type of machine as depicted in Table 3.

Table 3. Connection intensity of machine type.

\begin{tabular}{ccc}
\hline S.No. & Machine Type & Connection Intensity \\
\hline M1 & Bartack & 7 \\
M2 & Feed of arm & 14 \\
M3 & Flatlock & 21 \\
M4 & Overlock & 28 \\
M5 & Single needle lock stitch & 35 \\
\hline
\end{tabular}

\subsubsection{Seam Length (SMV/SAM)}

The longer stitch can provide better hold between two fabrics. The length of the seam is also directly proportional to the SAM. Hence, the numerical value to the length of the seam is provided on the basis of the SAM. This could be interpreted as connection intensity being directly proportional to the SAM as illustrated in Table 4.

Table 4. Connection intensity of seam length.

\begin{tabular}{ccc}
\hline S.No. & SAM (Minutes) & Connection Intensity \\
\hline M1 & Less than 0.20 & 3 \\
M2 & Between 0.20 to 0.50 & 6 \\
M3 & Between 0.50 to 0.80 & 9 \\
M4 & Between 0.80 to 1 & 12 \\
M5 & More than 1 & 15 \\
\hline
\end{tabular}

\subsubsection{Fabric Attributes}

There is a lot of subjective parameters that differentiate one fabric from another. The connection of two parts also depends on its attributes such as material composition and color or design. A stronger stitch can be achieved if two fabrics with a similar material composition are stitched together. Similarly, if some fabrics are stitched together, then it could give better aesthetic appeal, while other fabrics may not give the same appeal. A durable fabric can give a stronger connection. On the basis of the aforementioned discussion, a numeric value is provided for the connection intensity on the basis of fabric attributes as presented in Table 5 . 
Table 5. Connection intensity of fabric attributes.

\begin{tabular}{ccc}
\hline S.No. & Fabric Attributes & Connection Intensity \\
\hline F1 & Color/design & 4 \\
F2 & Composition & 8 \\
F3 & Durability & 12 \\
F4 & Any two attributes & 16 \\
F5 & All three attributes & 20 \\
\hline
\end{tabular}

If all the three fabric attributes-i.e., colour, composition, and durability-are achieved, then the numeric value will be the highest. For two attributes, the numeric value will be 16 .

\section{A Case Study of Garment Redesign}

The process of redesign varies from one organization to another. A Sweden-based redesign organization is selected to understand the application of remodularization. This selected Swedish organization performs the process of redesign at a larger scale through separate redesign division. A dedicated team of designers carries out the redesign activities in the warehouse. The activities of the redesign are also carried out through satellite centers where local inhabitant volunteers redesign old textile or garments products. All of the redesign products are sold under the 'Remake' brand name. The organization has launched a new store to sell redesigned garments. The team has a standard operating procedure for the complete redesign activities. However, at present, activities such as parts extraction and identification, determination, and the implementation of connections are done based on the memory, intuition, and experience of the designers. Figure 6 demonstrates the steps followed for redesign at the selected organization.

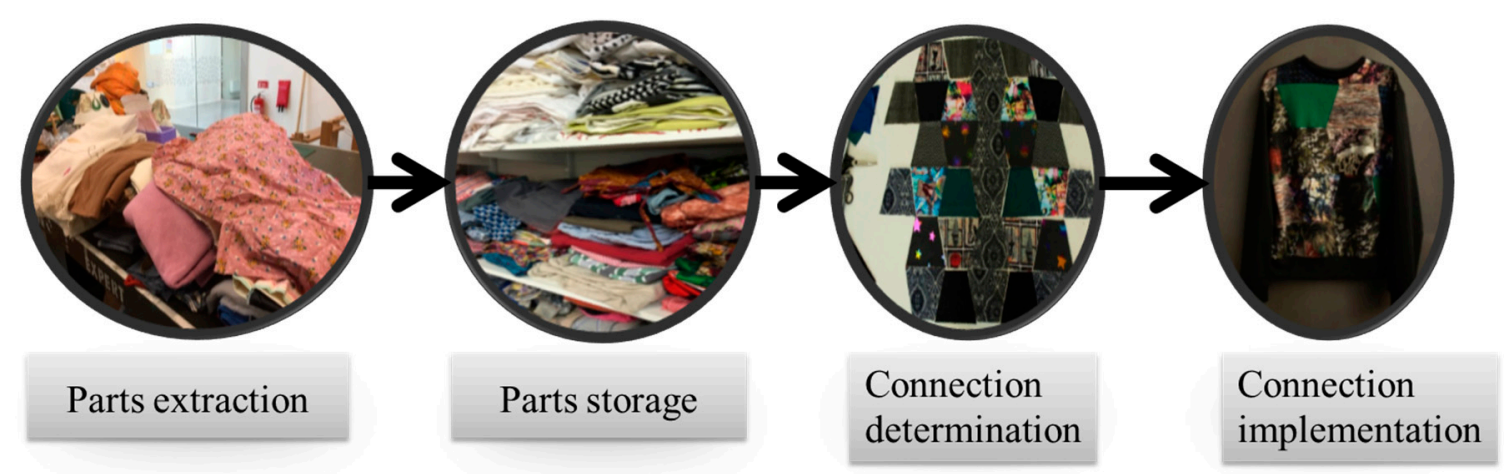

Figure 6. Stage of modularized garment redesign process (source: organization page on social media).

However, the above remodularizations model can be used for the redesign process. The steps can be elaborated as follows.

\subsection{Selection of Reference Products (Used Garments)}

Garments are selected on the basis of their aesthetic, functional qualities, perceived needs, labor-saving, and ease of reconstruction. Aesthetic qualities include style, the color, material composition, visual appeal, and prevalent demand. The functional qualities on the basis of which garments are selected vary from product to product. For example, denim jeans and jackets are selected on the basis of fabric sturdiness. Garments are generally discarded due to their worn or washed-out look. Interestingly, this feature of faded color or washed-out look increases its attractiveness and is considered to make a product more suitable for a redesign. T-shirts for men and women are also available in abundance in the collection of discarded garments. T-shirts and tops generally have little variations in the form of size, fabric, and constructions. Uniformity among the styles and the ease of 
handling of the fabrics make t-shirts and tops suitable for a redesign. Formal suits and long ladies' dresses are also considered appropriate for a redesign because of the higher quality and quantity of the usable fabrics. On the basis of the material usability and color/style forecast/trends of upcoming seasons, a 'wish list' is prepared by the redesign team. The durability of the material and disassembly effort is also considered during the preparation of a material 'wish list'. The sorting team picks the material as per wish the list, prepares a bag, and gives it to the redesign team after regular intervals.

\subsection{Extraction of Parts from Reference Products (Used Garments)}

The redesign process begins by deconstructing and analyzing the used products and associated materials for their best use and utilization. The redesign team observes each of the products considering the scope of redesign, and accordingly extracts a designable part/patch after disassembly. Basic garments such as t-shirts and ladies' dresses are cut to extract fabrics, while complex garments such as suits and jackets are converted to another form of garment. The products that are found suitable for the redesign are further sent for value-addition processes such as alteration, embroidery, and printing. The current organization has in-house facilities for basic embroidery and screen-printings. The part or patch of the fabrics and trims are stored separately on the racks as per the concept of remodularization. Each of the extracted parts is stored at different locations on the basis of their characteristics or future use. At present, the extraction and storage of parts are done manually. However, with the proposed remodularization model, the attributes information of the extracted parts can be stored in the computerized system. The type of attributes and the value for each of the parts can be entered in the remodularization system. Overall, each part can be physically categorized and stored on the basis of its attributes.

\subsection{Identification of Parts for Target Products (Redesigned Garments)}

The initial idea for the redesigning of garments is developed based on the demand forecast and the available extracted parts. Designs are finalized with the technique of draping and/or flat patternmaking. Subsequently, the parts search starts to achieve the desired aesthetic look. At present, this whole process of search is done manually and mainly on the basis of memory. The proposed remodularization model can facilitate an ease of search of the required parts. The parts requirements are raised based upon the size and styles of the intended redesigned garments. Each of the required parts has specific attributes and value associated with it. The parts are searched on the basis of their attributes and values. If a part with a particular attribute and value is not available in the parts database, then parts with similar characteristics can be selected to get the desired output after modification.

\subsection{Determination of Connections}

The next step in this process is a determination of connection for the selected parts. The selected parts are connected as per the design prototype to achieve the desired look and draping. Currently, the redesign organization establishes connections on a trial basis by making several permutations and combinations. The manual process of connection establishment is a time-consuming and tedious process. Some modifications are also done if the shape and size of the connecting seam are not similar. The feasibility of the transformations is also considered during the modification and reconstruction processes. The process of this connection establishment can be simplified with the help of the proposed remodularization model. The computerized remodularization model system can suggest suitable parts for connections. The modularized redesign system will keep on determining connections until the time where a suitable connection is not achieved.

\subsection{Calculation of Design Fitness for Connections}

Once parts are selected and connections are determined, then the next step is to implement connection. A good design can be achieved by proper stitching of the parts with the help of garment design and production knowledge. Under a few circumstances, proper connection constraints can 
be also required. For example, if the composition of the fabrics vary from each other, it is not easy to implement connections, even though the attributes and its value are the same. Understanding these nuances related to production constraints are gradually developed with experience. The redesign system will keep on doing the reconstruction process until the time where the desired perfection is not achieved. At the later stage, modification or reconstruction can be repeated if the designer is not satisfied with design or fitting. The calculation of design fitness is demonstrated in the next subsection by considering a basic t-shirt as an example.

\subsection{An Illustration of Design Fitness for Polo T-Shirt}

Step 1-2: Identification of target and selection of reference product

The below example has been selected to calculate the connection intensity i.e., the example $\mathrm{t}$-shirt/top is manufactured with extracted parts from used clothes. A t-shirt can be redesigned from two or more reference products. The targeted redesigned products could also be one, or more than one. Figure 7 depicts the reference and target garments for illustrative purposes.

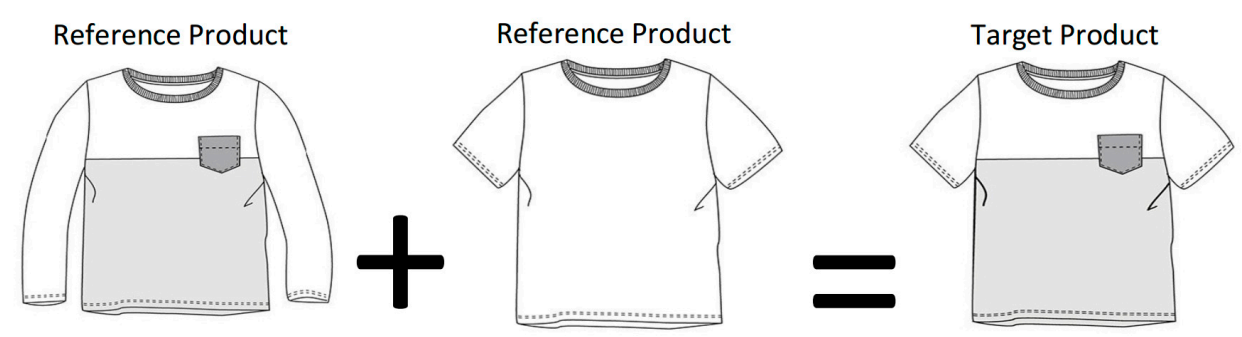

Figure 7. Extraction and determination of parts.

Step 3-4: Extraction and determination of parts

A basic t-shirt consists of five parts i.e., the front, back, sleeve, neckband, and sleeve rib; these parts can be extracted from different garments and can subsequently be identified for new redesigned garments. Figure 8 illustrates the process of part extraction and stitching for a t-shirt.

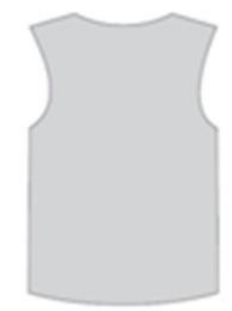

Front

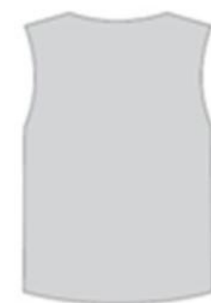

Back

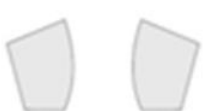

Sleeve

(a) Extraction of parts from used garments

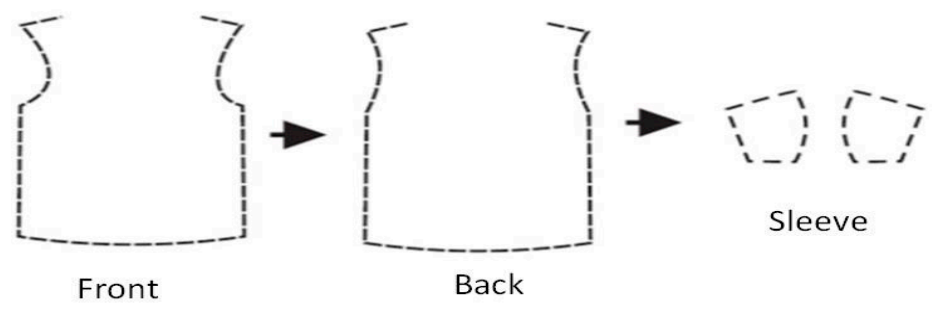

(b) Stitching of parts

Figure 8. Extraction and stitching of parts. 
Step 5-6: Calculation of connection intensity and design fitness

The connection intensity or design fitness can be calculated based on the operation required to stitch the parts to form a garment. The calculation of design fitness is done in Table 6 for the $t$-shirt according to rule formulated in Section 4.2.

Table 6. Calculation of design fitness for a t-shirt. SAM: standard allowed minute, CI: connection intensity.

\begin{tabular}{|c|c|c|c|c|c|c|}
\hline Module Connection & Operation & Seam & Machine & SAM & Fabric & Total CI \\
\hline \multirow{3}{*}{$\begin{array}{l}\text { Module } 1 \text { (front) and } \\
\text { Module } 2 \text { (back) } \\
\text { module }\end{array}$} & Shoulder attach & Superimposed (30) & OL (30) & $0.56(9)$ & All attributes (20) & 89 \\
\hline & Shoulder top stitch & Superimposed (30) & SNLS (35) & $0.34(6)$ & All attributes (20) & 91 \\
\hline & Side seam & Superimposed (30) & OL (30) & $2(15)$ & All attributes (20) & 95 \\
\hline \multirow{2}{*}{$\begin{array}{l}\text { Body and Module } 3 \\
\text { (sleeve) }\end{array}$} & Sleeve attach & Superimposed (30) & OL (30) & $0.89(12)$ & All attributes (20) & 92 \\
\hline & Sleeve top stitch & Superimposed (30) & SNLS (35) & $0.60(9)$ & All attributes (20) & 94 \\
\hline \multirow{2}{*}{ Module 4 (Rib attach) } & Neckband attach & Superimposed (30) & SNLS (35) & $0.77(12)$ & All attributes (20) & 97 \\
\hline & Sleeve rib attach & Superimposed (30) & SNLS (35) & $1.0(12)$ & All attributes (20) & 97 \\
\hline \multicolumn{6}{|c|}{ Cumulative average $\mathrm{CI}$ for garment } & 95 \\
\hline
\end{tabular}

Given the above conditions, the design fitness of the redesigned fitness for the $t$-shirt is calculated and was found to be 95 . This is relatively good, and the redesigned garments can be considered good for their use.

\subsection{Benefits of Modularized Redesign Model}

The direct reuse of a used or discarded product is sustainable compared to making a new product from raw materials. The process of collection, sorting, and the redistribution of apparel can save approximately $98 \%$ of the energy compared to manufacturing products from raw material [46]. However, there are various limitations of direct reuse, such as styles becoming outdated or some parts getting damaged. Therefore, redesign could be a sustainable option to modify old products as per the latest fashion trends. The good conditioned parts could be used by the remodularization model for a high-value redesign, while the defected parts could be a process of inferior use or material extraction. Paras and Curteza [47] in their study on reverse value chain decisions have found that upcycling or redesign are the most preferred alternatives, as the redesign process improves the value of existing products by increasing the usefulness of products for the consumer and making products resource-efficient. Figure 9 shows an apparel life cycle with different reverse supply chain alternatives such as reuse, manual redesign, genetic algorithm-based modular redesign, recycling, and waste incineration/landfill.

The life cycle assessment of a product is an important tool to analyze environmental damage such as resource depletion, energy consumption, and greenhouse emissions. Damage to the environment occurs in the process of raw material extraction, production, distribution, use, reuse, and disposal of the product. Reverse value chain alternatives such as reuse and redesign give a second life to a product with little or no environmental impacts, whereas process such as recycling for material extraction, incineration, and landfill have negative impacts on the environment due to energy consumption, pollutions, and landfill occupations. However, the direct reuse and manual redesign process have limitations in the terms of reuse and aesthetics. The genetic algorithm-based modular redesign process can overcome the limitation by proposing the best redesign alternatives through an iterative process. Thus, the existing product can be redesigned to be used for longer, and hence, the producess reduces resource depletion. The GA-based modular redesign alternatives for the reverse value chain for apparel can optimize the extraction of parts from discarded products. Hence, the amount of apparel that is meant for material recycling or landfill/incineration can be minimized. This will save the energy that is required to manufacture a product from the extracted raw material. The lower amount of landfill/incineration leads to a reduction in pollution and landfill occupations. Thus, it can be concluded on the basis of life cycle assessment (LCA) that GA-based modular redesign is an 
environmentally efficient reverse value chain alternative. The environmental impact of the proposed GA-based modular design can be further improved with a simplified life cycle assessment. The goal of a simplified life cycle approach is to reduce the product design complexity in the early design stage. This simplified LCA optimizes the extraction and reconstruction of parts by easing down disassembly efforts.

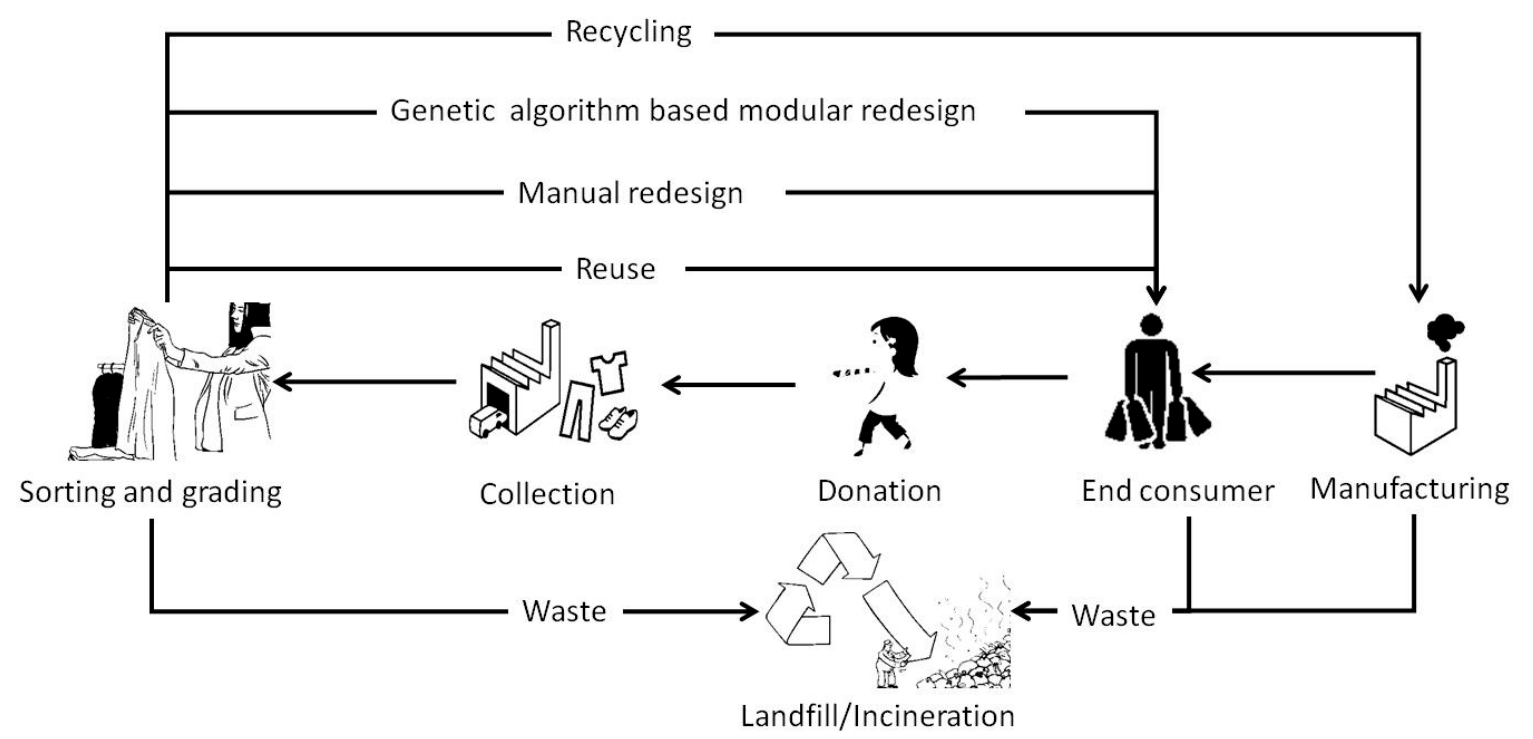

Figure 9. Lifecycle assessment of apparel with different reverse supply chain scenarios.

In order to further understand the benefits of the modularized redesign model over the manual redesign process, an analysis has been performed to compare the time consumed in each process. The time taken in the manual redesign process varies according to the complexity of the garments. Simple or basic garments such as ladies' knitted tops, $t$-shirts, and shirts are relatively easy to redesign. Almost one to two hours are consumed to redesign basic garments. Whereas for garments of medium complexity, such as trouser and jeans, the redesign process needs $6-8 \mathrm{~h}$. Jackets and ladies dresses are considered high-complex garments, and the redesign process requires 10-18 $\mathrm{h}$ (up to two working days). Most of these times these are incurred are during the redesign ideation and the determination of connections. The remodularization model can be applied to shorten the time taken for redesign ideation and the determination of connections. The remodularization model can perform ideation and connection determination tasks in a matter of minutes with the help of computerized information. A comparison of the time taken for the redesign process with the manual method and application of the remodularization model is depicted in the below pictures:

From Figure 10, it can be easily understood that the remodularization modal can save up to $75 \%$ of the time compared to a manual process. Apart from saving time, the proposed model also improves the quality of the redesigned products. During the manual redesign process, due to the limitation of the human brain, the suitable parts cannot be identified. Whereas the GA-based modular design can suggest the best suitable parts for a redesign. The above discussion summarizes the benefits of the remodularization model over the manual redesign process. In other words, this can also be considered as a recommendation to improve the current redesign process. 


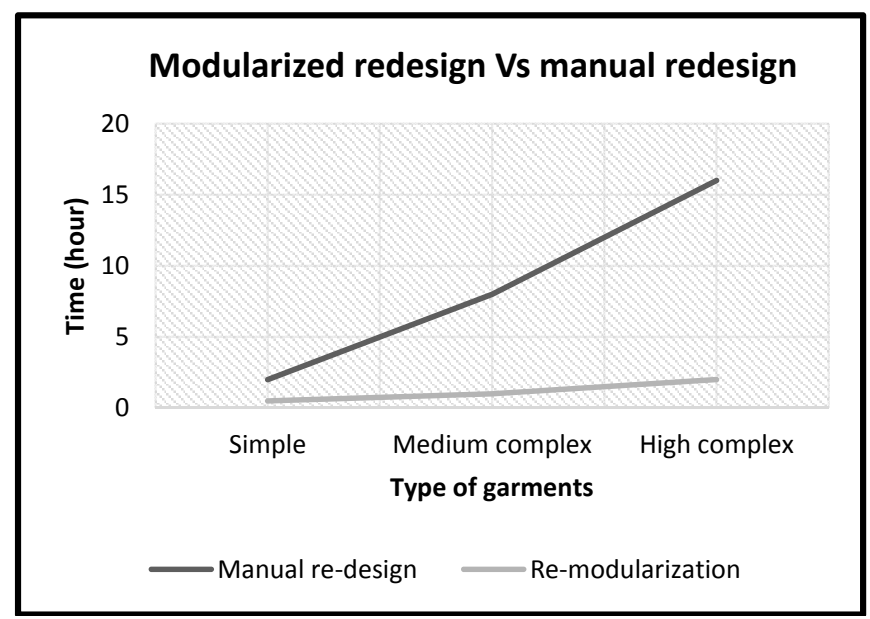

Figure 10. Benefits of the remodularization model over manual redesign.

\section{Discussion and Conclusions}

The redesign is an important part of product development to extend the lifespan and environmental impact of products. The research techniques need to be innovative in order to maximize the use of end-of-use or end-of-life products and fulfill customer requirements. The success of redesign is largely dependent on the product innovation, new technologies, resources, and skill that is used to achieve the desired target. This study introduces an innovative garment redesign system based on a modular and genetic algorithm, which shows that a well-planned design system enhances the chance of success for a redesigned product. The innovative design approach combines two or more distinct design products that increase the redesign quality and material utilization, and reduce the redesign time. The innovative redesign method based on a genetic algorithm and modular technique can be implemented to simplify the redesign process.

In this study, the garment is chosen as an illustrative product to understand the redesign process. Domain knowledge was used to assign a numerical value for the connection of two parts. Seam type (connection type), machine type, seam length (connection length), and fabric attributes are considered as parameters for the connection of garment parts. The maximum weight for the seam type, machine type, seam length, and fabric attributes are assigned $35 \%, 30 \%, 15 \%$, and $20 \%$, respectively. Depending on the strength of the connection, a lesser or maximum value for each type of connection attribute is provided. The design fitness for the garment is calculated by the average sum of all of the connections of a garment.

The feasibility of the redesign system is checked through the case study of a redesigned company based in Sweden. The results show that the application of the model could simplify the existing process of redesign. In the present scenario, all of the processes, right from parts extraction to reconstruction, are done manually on the basis of the expertise and skills of the designers. The application of modularized model can also enable others to perform redesign activities based on the redesign history. It has been found that application of a remodularization model can save significant time compared to the manual process. An illustration of design fitness is provided with the help of a t-shirt that is a reference as well as the target product. The similar function parts are extracted from the reference product for the target function in the example. However, the current method and proposed model can also work for the extraction of parts from different products. Therefore, the current research could be extended by applying the proposed redesign model for another industry.

The above-presented redesign model can provide an innovative and easier approach for the designers. The designers have suggested that basic products such as t-shirts/tops are easy to redesign as compared to complex products such as coat and ladies' dresses. In addition, designers have also acknowledged that durable products such as denim trousers are suitable for redesign. The model 
can be further used and verified in another industrial context. The practitioners can learn from the systematic redesign approach presented in the case study and illustration to simplify the redesign process. Future researchers can explore the possibility of integrating consumers with the redesign model proposed in this work by exploiting computer science and information technology knowledge.

Author Contributions: M.K.P., and L.W. conceived and designed the research. D.E., R.P., A.C., Y.C., and L.W. contributed to fine-tune the analysis and discussion. M.K.P., and L.W. wrote the paper.

Funding: The APC and corresponding research work were funded by the National Natural Science Foundation of China (Grant No. 61702352). This research was also funded by “Erasmus Mundus joint doctoral programme (SMDTex)"European Commission and the EU window of Chinese scholarship council. Engineering Research Center of Clothing of Zhejiang Province (Open Research Foundation Grant No. 2018FZKF01) has also partially aided the current research.

Conflicts of Interest: The authors declare no conflict of interest.

\section{References}

1. Chang, W.-C. Researching design trends for the redesign of product form. Des. Stud. 2003, 24, $173-180$. [CrossRef]

2. Luh, D.B. The development of psychological indexes for product design and the concepts for product phases. Des. Manag. J. 1994, 5, 30-39. [CrossRef]

3. Smith, S.; Smith, G.; Shen, Y.-T. Redesign for product innovation. Des. Stud. 2012, 33, 160-184. [CrossRef]

4. Li, Z.-S.; Kou, F.-H.; Cheng, X.-C.; Wang, T. Model-based product redesign. Ijcsns 2006, 6, 100-108.

5. Baxter, M. Product Design: A Practical Guide to Systematic Methods of Development of New Products; Nicolas Hayek: A Brief History of Swatch' [Video Recording]; Chapman \& Hall: London, UK, 1995.

6. Ma, J.; Kremer, G.E.O. A sustainable modular product design approach with key components and uncertain end-of-life strategy consideration. Int. J. Adv. Manuf. Technol. 2015, 85, 741-763. [CrossRef]

7. Kim, H.-S.; Cho, S.-B. Application of interactive genetic algorithm to fashion design. Eng. Appl. Artif. Intell. 2000, 13, 635-644. [CrossRef]

8. Pnueli, Y.; Zussman, E. Evaluating the end-of-life value of a product andimproving it by redesign. Int. J. Prod. Res. 1997, 35, 921-942. [CrossRef]

9. Bovea, M.; Wang, B. Redesign methodology for developing environmentally conscious products. Int. J. Prod. Res. 2007, 45, 4057-4072. [CrossRef]

10. Janz, D.; Sihn, W.; Warnecke, H.-J. Product redesign using value-oriented life cycle costing. CIRP Ann. Manuf. Technol. 2005, 54, 9-12. [CrossRef]

11. Yang, Q.; Yu, S.; Sekhari, A. A modular eco-design method for life cycle engineering based on redesign risk control. Int. J. Adv. Manuf. Technol. 2011, 56, 1215. [CrossRef]

12. Simpson, T.W.; Maier, J.R.; Mistree, F. Product platform design: Method and application. Res. Eng. Des. 2001, 13, 2-22. [CrossRef]

13. Alizon, F.; Shooter, S.B.; Simpson, T.W. Improving an existing product family based on commonality/diversity, modularity, and cost. Des. Stud. 2007, 28, 387-409. [CrossRef]

14. Sa'Ed, M.S. A methodology to redesign heterogeneous product portfolios as homogeneous product families. Comput. Aided Des. 2007, 39, 1065-1074.

15. Zhang, Y.; Gershenson, J.K. An Initial Study of Direct Relationships between Life-Cycle Modularity and Life-Cycle Cost. Concurr. Eng. 2003, 11, 121-128. [CrossRef]

16. Jose, A.; Tollenaere, M. Modular and platform methods for product family design: Literature analysis. J. Intell. Manuf. 2005, 16, 371-390. [CrossRef]

17. Lu, J.-M.; Wang, M.-J.J.; Chen, C.-W.; Wu, J.-H. The development of an intelligent system for customized clothing making. Expert Syst. Appl. 2010, 37, 799-803. [CrossRef]

18. Paras, M.K.; Pal, R. Application of Markov chain for LCA: A study on the clothes 'reuse'in Nordic countries. Int. J. Adv. Manuf. Technol. 2018, 94, 191-201. [CrossRef]

19. Wadhwa, S.; Bhoon, K.S.; Chan, F.T.S. Postponement strategies for re-engineering of automotive manufacturing: Knowledge-management implications. Int. J. Adv. Manuf. Technol. 2006, 39, 367-387. [CrossRef] 
20. Zhou, H.; Xu, Y.; Wang, L.; Chen, Y. A garment design method based on modularization. Text. Res. J. 2016, 86, 1710-1715. [CrossRef]

21. Liu, X. Research on product design knowledge reuse through linkage with design iteration records. In Proceedings of the IFIP International Conference on Network and Parallel Computing, NPC 2008, Shanghai, China, 18-21 October 2008; pp. 510-515.

22. Liu, X.; Wang, Y. Flexible case retrieval on the web for product design support. Mech. Sci. Technol. 2001, 7, 716-719.

23. Kasarda, M.E.; Terpenny, J.P.; Inman, D.; Precoda, K.R.; Jelesko, J.; Sahin, A.; Park, J. Design for adaptability (DFAD)_A new concept for achieving sustainable design. Robot. Comput. Integr. Manuf. 2007, 23, 727-734. [CrossRef]

24. Tseng, H.; Chang, C.; Li, J. Modular design to support green life-cycle engineering. Expert Syst. Appl. 2008, 34, 2524-2537. [CrossRef]

25. Kurniawan, H.; Sofianti, T.D.; Pratama, A.T.; Tanaya, P.I. Optimizing Production Scheduling Using Genetic Algorithm in Textile Factory. J. Syst. Manag. Sci. 2014, 4, 27-44.

26. Kargar, M.; Payvandy, P. Optimization of fabric layout by using imperialist competitive algorithm. J. Text. Polym. 2015, 3, 55-63.

27. Fischer, A.D.; Dagli, C.H. Employing subgroup evolution for irregular-shape nesting. J. Intell. Manuf. 2004, 15, 187-199. [CrossRef]

28. Junior, B.A.; Pinheiro, P.R.; Saraiva, R.D. A Hybrid Methodology for Nesting Irregular Shapes: Case Study on a Textile Industry*. IFAC Proc. Vol. 2013, 46, 15-20. [CrossRef]

29. Leo Ho Wai, Y.; Wallace, K.S.T. A hybrid genetic approach for garment cutting in the clothing industry. IEEE Trans. Ind. Electron. 2003, 50, 449-455. [CrossRef]

30. Wong, W.K.; Leung, S.Y.S. A Hybrid Planning Process for Improving Fabric Utilization. Text. Res. J. 2009, 79, 1680-1695. [CrossRef]

31. Yang, H.-H.; Lin, C.-L. On genetic algorithms for shoe making nesting-A Taiwan case. Expert Syst. Appl. 2009, 36, 1134-1141. [CrossRef]

32. Farrell, R.S.; Simpson, T.W. Improving cost effectiveness in an existing product line using component product platforms. Int. J. Prod. Res. 2010, 48, 3299-3317. [CrossRef]

33. Mutingi, M.; Dube, P.; Mbohwa, C. A Modular Product Design Approach for Sustainable Manufacturing in A Fuzzy Environment. Procedia Manuf. 2017, 8, 471-478. [CrossRef]

34. Gu, P.; Hashemian, M.; Sosale, S.; Rivin, E. An integrated modular design methodology for life-cycle engineering. CIRP Ann. 1997, 46, 71-74. [CrossRef]

35. Falkenauer, E. Genetic Algorithms and Grouping Problems; Wiley: New York, NY, USA, 1998.

36. De Lit, P.; Falkenauer, E.; Delchambre, A. Grouping genetic algorithms: An efficient method to solve the cell formation problem. Math. Comput. Simul. 2000, 51, 257-271. [CrossRef]

37. Lapolla, K.; Sanders, E.B.N. Using Cocreation to Engage Everyday Creativity in Reusing and Repairing Apparel. Cloth. Text. Res. J. 2015, 33, 183-198. [CrossRef]

38. Paras, M.K.; Pal, R.; Ekwall, D. Systematic literature review to develop a conceptual framework for a reuse-based clothing value chain. Int. Rev. Retail. Distrib. Consum. Res. 2018, 28, 231-258. [CrossRef]

39. Janigo, K.A.; Wu, J. Collaborative redesign of used clothes as a sustainable fashion solution and potential business opportunity. Fash. Pract. 2015, 7, 75-98.

40. Paras, M.K.; Curteza, A. Revisiting upcycling phenomena: A concept in clothing industry. Res. J. Text. Appar. 2018, 22, 46-58. [CrossRef]

41. Earley, R.; Reitan Andersen, K. Design Thinking for Sustainability: A Case Study of a Research Project between Hennes \& Mauritz and Textiles Environment Design. In Proceedings of the 20th Annual International Sustainable Developm Ent Research Conference Nce Norwegian University of Science and Technology, Trondheim, Norway, 18-20 June 2014.

42. Keith, S.; Silies, M. New life luxury: Upcycled Scottish heritage textiles. Int. J. Retail. Distrib. Manag. 2015, 43, 1051-1064. [CrossRef]

43. Paras, M.; Ekwall, D.; Pal, R.; Curteza, A.; Chen, Y.; Wang, L. An Exploratory Study of Swedish Charities to Develop a Model for the Reuse-Based Clothing Value Chain. Sustainability 2018, 10, 1176. [CrossRef]

44. Deschamps, T.C.; Carnie, B.; Mao, N. Public consciousness and willingness to embrace ethical consumption of textile products in Mexico. Text. Cloth. Sustain. 2016, 2, 6. [CrossRef] 
45. Das, S.K.; Yedlarajiah, P.; Narendra, R. An approach for estimating the end-of-life product disassembly effort and cost. Int. J. Prod. Res. 2000, 38, 657-673. [CrossRef]

46. Woolridge, A.C.; Ward, G.D.; Phillips, P.S.; Collins, M.; Gandy, S. Life cycle assessment for reuse/recycling of donated waste textiles compared to use of virgin material: An UK energy saving perspective. Resour. Conserv. Recycl. 2006, 46, 94-103. [CrossRef]

47. Paras, M.K.; Curteza, A. Application of fuzzy technique for closed loop decision in clothing value chain. Int. J. Value Chain Manag. 2018, 9, 105-121. [CrossRef]

(C) 2018 by the authors. Licensee MDPI, Basel, Switzerland. This article is an open access article distributed under the terms and conditions of the Creative Commons Attribution (CC BY) license (http:/ / creativecommons.org/licenses/by/4.0/). 